\title{
Kindler Syndrome: A Rare Genodermatosis Presenting in 2 Brothers
}

\author{
Ryan Fischer ${ }^{2 *}$, Muhammad Imran ${ }^{1}$, Anand Rajpara ${ }^{2}$, Joseph Blackmon² \\ ${ }^{1}$ University of Kansas Medical Center, Department of Medicine, Division of Allergy, Clinical Immunology and Rheumatology, \\ Kansas City, KS \\ ${ }^{2}$ University of Kansas Medical Center, Department of Medicine, Division of Dermatology, Kansas City, KS
}

*Corresponding author: Ryan Fischer, MD, PGY-4 Dermatology Resident, Department of Dermatology, 3901 Rainbow Boulevard, Kansas City, Kansas 66160, Tel: (913) 588-5000; E-mail: ryanfischeruk@gmail.com, rfischer3@kumc.edu

Citation: Fischer, R., et al. Kindler Syndrome: A Rare Genodermatosis Presenting in 2 Brothers. (2016) Cell Immunol Serum Biol 2(1): 29- 31 .

Received Date: August 27, 2015

Accepted Date: April 26, 2016

Published Date: May 02, 2016

DOI: $10.15436 / 2471-5891.16 .002$

\section{Case Report}

We report a case of two Asian brothers, aged 21 and 29, born to consanguineous parents, who presented with cutaneous hyper- and hypopigmentation, skin atrophy, and skin fragility of the face, neck, and distal parts of the extremities. They were the first and second, full-term pregnancies in a family without any history of skin disease. Both patients had a history of recurrent minor trauma-induced blistering over the extensor aspects of the forearms, lower legs, dorsal hands, and feet since infancy. The blisters contained either serous or hemorrhagic fluid and typically ruptured within 4 to 5 days producing erosions that healed with dyspigmentation and atrophy, but no scarring. The tendency for blister formation after minor trauma gradually subsided by the ages of 13 and 15, respectively. Additionally, the brothers had severe skin fragility affecting the face, neck, distal limbs, and gums. They reported increased photosensitivity with sunburn after minimal sun exposure and exacerbation of their disease during the summer months. Dysphagia started at the ages of 17 and 19 and has been persistent with variable severity. The evolution of the disease led to the subsequent development of reticular pigmentation, progressive skin atrophy, gingival fragility with bleeding, webbing of the fingers and toes, nail dystrophy, dysuria and eye soreness.

On physical examination, both patients had normal physical and mental development. Skin examination revealed poikiloderma, patchy hyperpigmentation and hypopigmentation, and telangiectasias (Figures 1 and 2). Also, skin fragility was noted mainly on the face, neck, upper chest, and over the dorsal hands and feet.

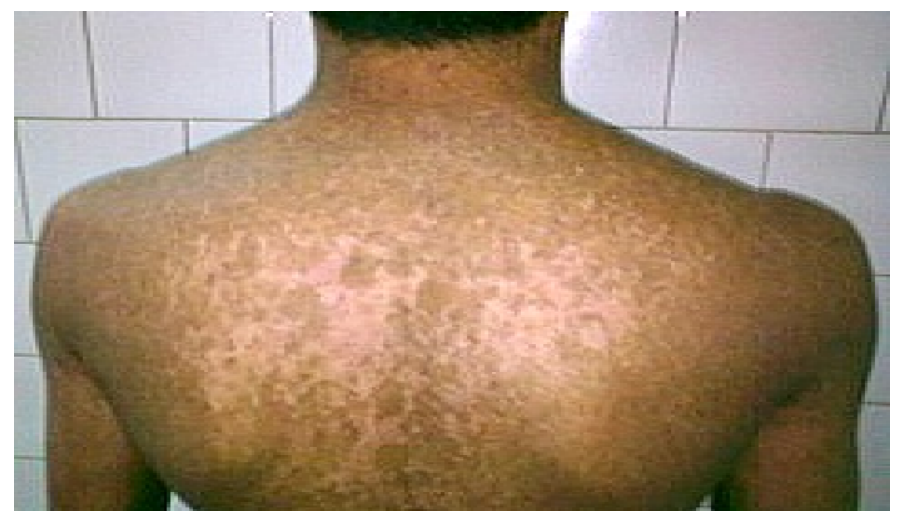

Figure 1: Patchy hypo- and hyperpigmentation with poikiloderma over the back

Copyrights: (C) 2016 Fischer, R. This is an Open access article distributed under the terms of Creative Commons Attribution 4.0 International License. 


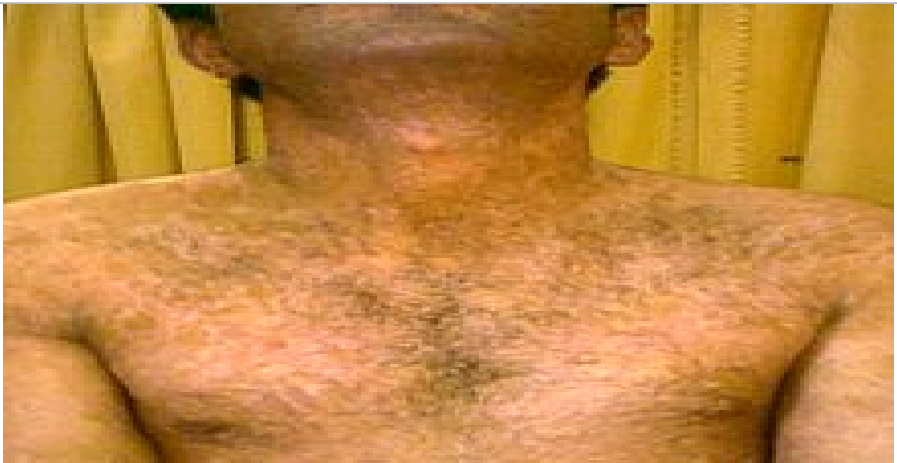

Figure 2: Patchy hyperpigmentation with poikiloderma on the upper chest and neck

There was no evidence of scarring. Most of the nails were rough, discolored and showed longitudinal ridging (Figure 3). Dermatoglyphic patterns were lost on the palms and soles (Figure 4). Examination of the oral cavity showed poor dentition, gingival swelling with easy bleeding, hyperpigmentation of the lips, and synechiae between the lips and the gums (Figure 5).

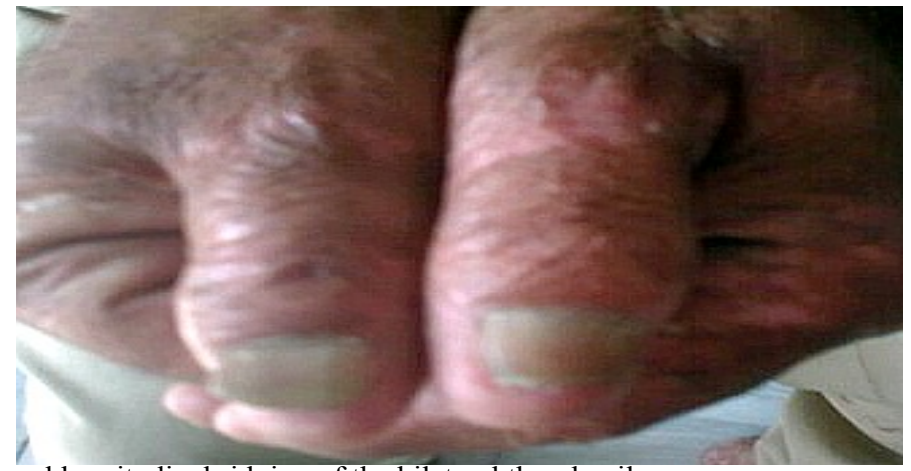

Figure 3: Yellowish discoloration and longitudinal ridging of the bilateral thumbnails

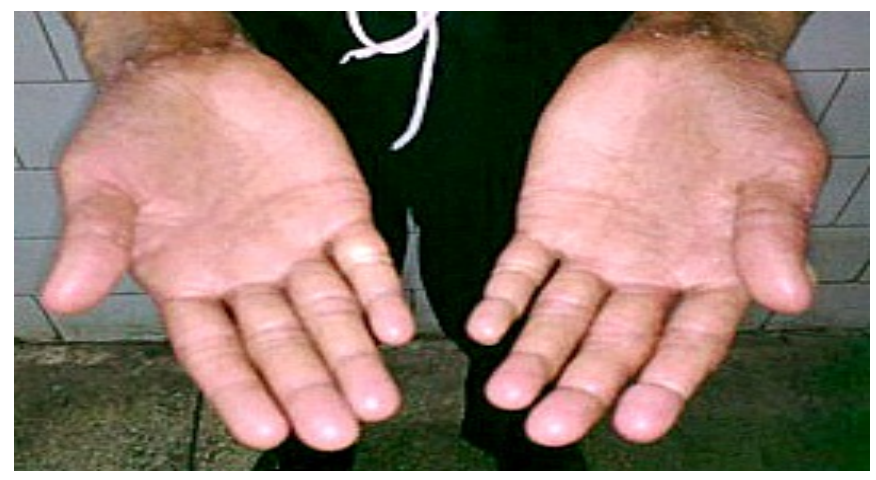

Figure 4: Loss of dermatoglyphic pattern on the palms

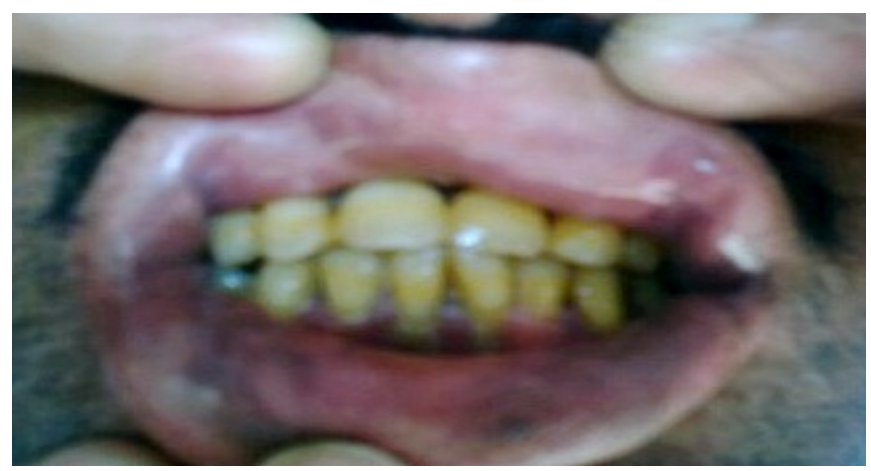

Figure 5: Hyperpigmentation of the mucosal lips with gingival swelling

Ophthalmologic evaluation revealed sparse eyelashes and ectropion of the lower eyelids. Blood tests, urine analysis and stool examinations were normal. An EGD performed on one of the brother's demonstrated esophageal stenosis, which was subsequently surgically corrected.

Skin biopsies demonstrated epidermal atrophy, flattening of the rete ridges, basal layer focal vacuolization and degener- 
ation, capillary dilatation (telangiectasis), pigmentary incontinence and papillary dermal edema. The patient could not afford the genetic analysis. On the basis of history, physical exam and histopathology, a diagnosis of Kindler syndrome was made. Treatment was mainly symptomatic and preventative with a focus on trauma avoidance, sun avoidance, sun protection, and wound care.

\section{Discussion}

Initially described by Theresa Kindler in $1954^{[1]}$, Kindler syndrome is a rare autosomal-recessive genodermatosis ${ }^{[2]}$. Kindler syndrome is a chronic blistering disorder that often presents initially with trauma-induced blisters in the congenital or neonatal period. Blisters occur primarily on the dorsa of the hands and feet and the tendency to blister decreases as the patient ages. Patients also experience photosensitivity that decreases with age. In older patients with Kindler syndrome, a prominent clinical feature is poikilodermatous skin change. These changes are usually apparent by around age five and then persist throughout the remainder of life. Another common clinical manifestation of Kindler syndrome is diffuse cutaneous atrophy ${ }^{[3]}$. Other less common manifestations include palmoplantar hyperkeratosis ${ }^{[1,4]}$, nail dystrophy ${ }^{[3,4]}$, webbing of the fingers and toes ${ }^{[3]}$, and mucous membrane involvement, namely stenosis of the esophagus, anus, and/or urethral meatus ${ }^{[3,4]}$.

In 2003, a loss-of-function mutation was mapped to a gene on 20p12.3 and named KIND1 (encoding kindlin-1). The KIND1 (also referred to as FERMT1) mutation was discovered in an analysis of a 26 patient cohort of a Native American tribe from Panama. Kindlin-1 is a signaling protein involved in actin cytoskeleton linking to the extracellular matrix. Kindler syndrome is the first skin fragility disorder known to involve the extracellular matrix binding to actin, instead of keratin ${ }^{[5]}$.

Skin biopsy specimens of patients with Kindler syndrome usually demonstrate poikilodermatous change, epidermal atrophy, and cleavage of either the basal layer or the lamina lucida ${ }^{[6]}$. Other characteristic pathology that can be seen includes marked reduplication of the lamina densa and cleft formation in disrupted areas of lamina densa ${ }^{[7]}$. Immunohistochemically, Kindler syndrome can be tested for by presence of anti-kindlin-1 antibodies, with levels usually markedly reduced or absent, but levels are not always reduced ${ }^{[8]}$. In addition, since Kindler syndrome presents similarly to dystrophic epidermolysis bullosa, it is worth noting that sequencing the type VII collagen (COL7A1) gene will show no mutations in patients with Kindler syndrome.

Differential diagnosis mainly includes dystrophic epidermolysis bullosa and poikiloderma congenitale, as Kindler syndrome has features of both; cutaneous atrophy and acral blister distribution are two observations that would favor Kindler syndrome. Several pre-malignant and malignant conditions have been observed in Kindler syndrome, namely actinic keratoses ${ }^{[6]}$, squamous cell carcinoma of the $\mathrm{lip}^{[9]}$ and hard palate ${ }^{[2]}$ and transitional cell carcinoma of the bladder ${ }^{[9]}$. There is an increased risk of squamous cell carcinoma in Kindler syndrome, and screening is recommended ${ }^{[2]}$.

Management of Kindler syndrome is mainly supportive and preventative. Trauma should be avoided to reduce blister formation and avoidance of sun may help with poikiloderma. Excellent wound care should be practiced to avoid infections of bullae. Since patient with Kindler syndrome are prone to esophageal, anal, and urethral stenosis, surgical procedures may be necessary to correct these abnormalities ${ }^{[2]}$.

Conflict of Interest: No conflict of interest or financial obligations

\section{References}

1. Kindler, T. Congenital poikiloderma with traumatic bulla formation and progressive cutaneous atrophy. (1954) Br J Dermatol 66(3): 104-111.

2. Jobard, F., Bouadjar, B., Caux, F., et al. Identification of mutations in a new gene encoding a FERM family protein with a pleckstrin homology domain in Kindler syndrome. (2003) Hum Mol Genet 12(8): 925-935.

3. Haber, R.M., Hanna, W.M. Kindler syndrome: Clinical and ultrastructural findings. (1996) Arch Dermatol 132(12): 1487-1490.

4. Draznin, M.B., Esterly, N.B., Fretzin, D.F. Congenital poikiloderma with features of hereditary acrokeratotic poikiloderma. (1978) Arch Dermatol 114(8): 1207-1210.

5. Forman, A.B., Prendiville, J.S., Esterly, N.B., et al. Kindler syndrome: report of two cases and review of the literature. (1989) Pediatr Dermatol 6(2): 91-101.

6. Shimizu, H., Sato, M., Ban, M., et al. Immunohistochemical, ultrastructural and molecular features of Kindler syndrome distinguish it from dystrophic epidermolysis bullosa. (1997) Arch Dermatol 133(90): 1111-1117.

7. Lai-Cheong, J.E., Ussar, S., Arita, K. Colocalization of kindlin-1, kindlin-2, and migfilin at keratinocyte focal adhesion and relevance to the pathophysiology of Kindler syndrome. (2008) J Invest Dermatol 128(9): 2156-2165.

8. Lotem, M., Raben, M., Zeltser, R., et al. Kindler syndrome complicated by squamous cell carcinoma of the hard palate: successful treatment with high dose radiation therapy and granulocyte-macrophage-colony-stimulating factor. (2001) Br J Dermatol 144(6): $1284-1286$.

9. Alper, J.C., Baden, H.P., Goldsmith, L.A. Kindler's syndrome. (1978) Arch Dermatol 114(3): 457.

10. Lai-Cheong, J.E., McGrath, J.A. Kindler Syndrome. (2010) Dermatol Clin 28(1): 119-124.

Ommega Online Publisher

Cellular Immunology and Serum Biology

Short Title : Cell Immunol Serum Biol
ISSN No: 2471-5891

E-mail: immunology@ommegaonline.com

website: www.ommegaonline.org 